\title{
Hypermethylation of the SPARC promoter and its prognostic value for prostate cancer
}

\author{
TIESHI LIU ${ }^{1,2}$, XUEFENG QIU ${ }^{2}$, XIAOZHI ZHAO ${ }^{2}$, RONG YANG $^{2}$, \\ HUIBO LIAN $^{2}$, FENG QU $^{2}$, XIAOGONG LI ${ }^{2}$ and HONGQIAN GUO ${ }^{1,2}$ \\ ${ }^{1}$ Department of Urology, Drum Tower Clinical Medical College of Nanjing Medical University, \\ Nanjing, Jiangsu 210008; ${ }^{2}$ Department of Urology, Nanjing Drum Tower Hospital, \\ The Affiliated Hospital of Nanjing University Medical School, Nanjing, Jiangsu 210008, P.R. China
}

Received July 11, 2017; Accepted November 15, 2017

DOI: 10.3892/or.2017.6121

\begin{abstract}
Secreted protein acidic and rich in cysteine (SPARC) is a secreted matricellular glycoprotein and plays a key role in the development of many tissues and organ types. However, the role of SPARC in prostate cancer (PCa) is still controversial. The aim of the present study was to investigate the abnormalities in the expression of SPARC and its promoter hypermethylation in prostate cancers and in correlated clinicopathological profiles. We examined the hypermethylation of the SPARC promoter as a potential mechanism for suppressing SPARC in PCa. The clinicopathological correlation between SPARC and its promoter expression and the prognostic significance of the aberrantly expressed genes were evaluated to identify novel biomarkers of PCa. SPARC expression was decreased in PCa cell lines, which correlated with hypermethylation of the SPARC promoter. Treatment with the demethylating agent 5-Aza-Cdr restored SPARC expression. Seventy percent (145 of 207) of the primary tumors exhibited SPARC hypermethylation, while only $2.6 \%$ was found in normal prostate mucosa $(n=38)$. In PCa cases, SPARC hypermethylation was correlated with a poorer prognosis $(\mathrm{P}=0.005$; relative risk 2.659, 95\% CI, 1.433-4.562). Our findings revealed potential diagnostic markers of PCa based on specific hypermethylated $\mathrm{CpG}$ sites and also provided new insights of SPARC as a novel biomarker and/or treatment modality for prostate cancer.
\end{abstract}

\section{Introduction}

Prostate cancer (PCa) is the most common malignancy in men, with an estimated 220,800 new cases and 27,540 deaths diagnosed in the USA in 2015 (1). Based on recent statistics, 161,360

Correspondence to: Dr Hongqian Guo, Department of Urology, Nanjing Drum Tower Hospital, The Affiliated Hospital of Nanjing University Medical School, Nanjing, Jiangsu 210008, P.R. China E-mail: dr_guohq@126.com

Key words: prostate cancer, SPARC, hypermethylation, biomarker newly diagnosed cases of PCa were reported. Among these cases, 26,730 American males were estimated to succumb to this disease in 2017, thus making PCa the most serious health problem among male patients (2). Radical prostatectomy and radiation therapy are the first choice treatments for $\mathrm{PCa}$, while the prognosis is different according to the clinical stage, pathological grading and individual differences. Prostate-specific antigen (PSA), has been clinically and widely used for the diagnosis of PCa. However, PSA detection presents a high false-positive rate, thus failing to accurately assess the malignancy grade of the tumor. Therefore, it does not fully satisfy the clinician's need to determine the treatment modality for patients with PCa (3). Thus, more specific molecular markers for PCa diagnosis and treatment are urgently needed.

Recently, cumulative evidence has indicated that the initiation and progression of PCa results from the accumulation of genetic and epigenetic changes (4,5). DNA methylation occurs in the early process of PCa and it has the potential to be a novel marker with high detection sensitivity $(6,7)$. Many genes, which play important roles in the pathogenesis and metastasis of PCa, are methylated. These methylated genes are potential biomarkers for the diagnosis and prognosis evaluation of PCa (8-10).

Secreted protein acidic and rich in cysteine (SPARC), also designated as osteonectin and BM-40, is expressed in tissues undergoing remodeling or repair and it also plays an important role in normal development. The level of SPARC expression depends on tumor types and the tumor cell environment. Different levels of SPARC expression have been reported in several tumors (11). Previous studies have revealed that SPARC can suppress PCa pathogenesis (12) and progression (13). Another study has demonstrated that exogenous SPARC inhibited the proliferation and migration of $\mathrm{PCa}$ cells (14). Furthermore, previous studies have revealed that SPARC hypermethylation was found in several cancers as well as PCas (15-18).

The purpose of the present study was to investigate the status of SPARC hypermethylation in PCa cell lines and tumor tissues and to investigate its clinical relevance to prostate cancers, as well as in patient outcome. Furthermore, the possibility of SPARC hypermethylation as a predictive biomarker in PCa was affirmed. 


\section{Materials and methods}

Cell culture. Five human PCa cell lines DU145, LNCaP, PC-3, NPC-3 and BPC-3, as well as the control prostate epithelial cell line (RWPE-1) were obtained from the Cancer Hospital of the Chinese Academy of Medical Sciences (Beijing, China). These cells were grown in Dulbecco's modified Eagle's medium (DMEM) supplemented with $10 \%$ fetal bovine serum (FBS) (both were obtained from Invitrogen Life Technologies Inc., Rockville, MD, USA) and incubated in $5 \% \mathrm{CO}_{2}$ at $37^{\circ} \mathrm{C}$.

Patient tissue specimens. A total of 207 surgically resected prostate samples were obtained from the Nanjing Drum Tower Hospital (Nanjing, China) from August 2005 to August 2007. The tissue samples were obtained and stored in liquid nitrogen immediately after being resected in the operating room. Informed consents were signed by all patients before surgery. All the clinical procedures were approved by the Medical Ethics Committee of the Nanjing Drum Tower Hospital. Two experienced radiologists ( $>10$ years of experience in urogenital radiology) reviewed the magnetic resonance imaging (MRI) images together and concorantly identified the suspicious lesions using the Prostate Imaging Reporting and Data System (PI-RADS) version 2.

Quantitative real-time, reverse transcription-polymerase chain reaction $(R T-P C R)$. Total RNA (1-2 $\mu \mathrm{g})$ was reverse transcribed using a SuperScript Pre-Amplification kit (Invitrogen Life Technologies, Carlsbad, CA, USA). Fast SYBR-Green Master Mix (Applied Biosystems; Thermo Fisher Scientific, Inc., Waltham, MA, USA) was used for the qPCR assay. The primer sequences as well as annealing temperatures and cycle numbers were derived from published studies $(19,20)$. The relative mRNA expression levels were normalized to the expression level of GAPDH using the $2^{-\Delta \Delta \mathrm{Ct}}$ method.

Immunoblot analysis. Total cell lysates were prepared and analyzed by immunoblot as aforementioned (19). The antibodies to SPARC (sc-25574, 1:800 dilution, rabbit polyclonal antibody; Santa Cruz Biotechnology, Inc., Santa Cruz, CA, USA) and GAPDH (sc-69778, 1:800 dilution, mouse monoclonal antibody; Santa Cruz Biotechnology) were used to detect SPARC and GAPDH, respectively. The labeling was detected with the SuperSignal West Dura Extended Duration Substrate (no. 37071; Thermo Fisher Scientific). The measurements were performed using the Odyssey Infrared Imaging System (LI-COR Biosciences, Lincoln, NE, USA).

Methylation specific PCR (MSP). DNA was isolated from the cell lines and the tissue sections using DNAzol reagent (Invitrogen Life Technologies) according to the manufacturer's protocol. The process of bisulfite conversion of the DNA samples $(1 \mu \mathrm{g})$ was conducted as previously described (21). The methylation status of the SPARC gene was determined using MSP (22). The primer sequences as well as the annealing temperatures and cycle numbers were derived from a published study (23).

Immunohistochemistry. Immunohistochemistry staining was performed on paraffin-embedded material. Sections
(4 $\mu \mathrm{m})$ were deparaffinised and subjected to antigen retrieval at $100^{\circ} \mathrm{C}$ for $20 \mathrm{~min}$ in $1 \mathrm{mmol} / 1$ EDTA buffer $(\mathrm{pH} 8.0)$ in a microwave oven. The sections were incubated at $4^{\circ} \mathrm{C}$ overnight with an anti-SPARC polyclonal antibody (1:100 dilution). The labeling was detected with the Envision Plus Detection kit (Dako, Carpinteria, CA, USA) and all sections were counterstained with hematoxylin. The extent of immunolabeling of SPARC was scored as follows: $0 \%$, negative; $\leq 10 \%$, focal; and $>10 \%$, positive. The negative group consisted of cancer cells with no detectable (-) or only trace staining of SPARC immunoreactivity (+). The positive group consisted of cancer cells with moderate (++) or high levels (+++) of SPARC immunoreactivity. Two independent histopathologists were blindly assigned to review the slides and score the staining.

5-Aza-Cdr treatment. In total, five PCa cell lines (DU145, PC-3, NPC-3, BPC-3 and LNCAP) at 65\% confluency were treated with the global genomic DNA demethylating agent 5-Aza-CdR. The cells were seeded in 6-well plates and incubated overnight in growth media. The normal growth media were replaced with growth media supplemented with $5 \mu \mathrm{M}$ 5-Aza-CdR and were replenished daily for 5 days. At the conclusion of the treatment, the cells were harvested for RNA, genomic DNA and protein as previously described. At least three independent experiments were performed.

Cell proliferation assay. The cell proliferation assay was carried out as previously described (17). Two cell lines (DU145 and PC-3) were seeded into 48 -well plates at a density of $1 \times 10^{4}$ cells/well. These cells were cultured in growth media supplemented with $5 \mu \mathrm{M}$ 5-Aza-CdR for 3 days. The cells were counted after treatment for 24,48 and $72 \mathrm{~h}$. A minimum of three independent experiments were performed. The cells in the normal growth media were treated as the controls.

Cell invasion and migration assays. CytoSelect 24-well cell migration and invasion assay kits (Cell Biolabs, Inc., San Diego, CA, USA) were used for the migration and invasion assays, according to the manufacturer's instructions. The cells ( $1 \times 10^{5}$ cells $/ \mathrm{ml}$ serum-free media with $5 \mu \mathrm{M} 5$-Aza-CdR) were added to the upper compartment of the chamber and the lower chambers were filled with $750 \mu \mathrm{l}$ media with $10 \%$ FBS. The cells were incubated for $24 \mathrm{~h}$ at $37^{\circ} \mathrm{C}$ and $5 \% \mathrm{CO}_{2}$, and then the non-migrated/non-invading cells on the upper side of the membrane were removed with a cotton swab. Migrated/ invaded cells on the lower sides of the inserts were fixed with methanol and stained with $0.1 \%$ crystal violet. The number of cells was counted in five random fields using optical microscopy. A minimum of three independent experiments were performed.

Statistical analysis. SPSS version 18.0 software (SPSS, Inc., Chicago, IL, USA) was used for statistical analysis. Continuous variables are shown as the mean \pm standard deviation (SD) and the differences between groups were evaluated using unpaired Student's t-tests. Statistical differences between groups were examined using Fisher's exact test, $\chi^{2}$ test and the Mann-Whitney test. Overall survival was evaluated using the Kaplan-Meier method and the log-rank test. $\mathrm{P}<0.05$ was considered to indicate a statistically significant difference. 
A

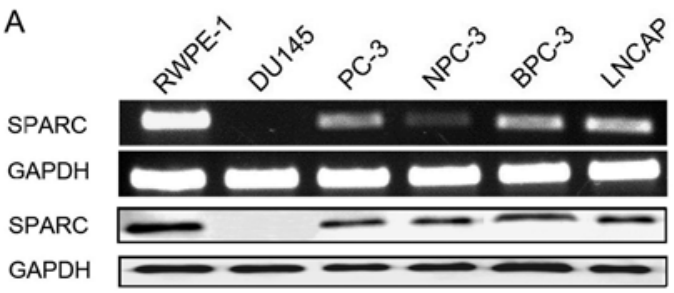

B

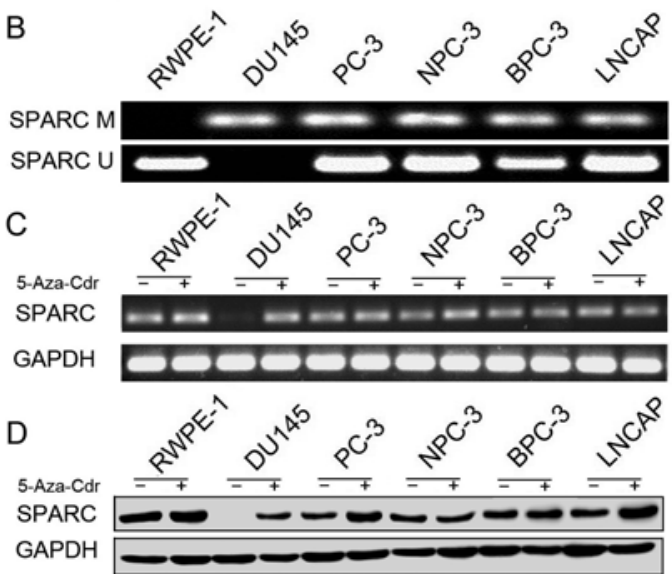

Figure 1. (A) SPARC mRNA (upper panel) and protein (lower panel) expression in RWPE-1 cells and five PCa cell lines. (B) Representative examples of MSP of the promoter in cell lines. The PCR products in the lanes $U$ and $\mathrm{M}$ indicate the presence of unmethylated and methylated templates, respectively. (C) SPARC mRNA expression in PCa cell lines after treatment with 5-Aza-Cdr. (D) SPARC protein expression in PCa cell lines after treatment with 5-Aza-Cdr. SPARC, secreted protein acidic and rich in cysteine; $\mathrm{PCa}$, prostate cancer; MSP, methylation specific PCR.

\section{Results}

Expression of SPARC and its hypermethylation analysis in PCa cell lines. We investigated the SPARC mRNA and protein expression in five prostate cancer cell lines and the control prostate epithelial cell line (RWPE-1). RT-PCR and immunoblotting revealed that SPARC was undetectable in the DU145 cell line and exhibited a low expression in the other four cancer cell lines (PC-3, NPC-3, BPC-3 and LNCAP; Fig. 1A). The expression levels of SPARC mRNA and protein in the RWPE-1 cell line were higher than in the PCa cell lines (Fig. 2A and B). Hypermethylation of the SPARC promoter was found in all five $(5$ out of $5,100 \%)$ prostate cancer cell lines (Fig. 1B). SPARC mRNA expression and hypermethylation of SPARC could not be detected in the DU145 cell line, but both were clearly exhibited in the other four cell lines. These results are similar to the data previously described in PCa cell lines (15).

Rescue of SPARC expression by 5-Aza-Cdr treatment. In order to confirm that the hypermethylation of the SPARC gene results in the loss of the SPARC protein expression, the prostate cells lines were cultured with 5-Aza-Cdr. The results indicated that the 5-Aza-Cdr treatment rescued SPARC mRNA and protein expression in the DU145 cell line where the expression of SPARC was lost. Furthermore, the demethylating agent increased the expression of SPARC mRNA and protein in the other four cancer cell lines (Figs. 1C and D and $2 \mathrm{C}$ and $\mathrm{D})$.
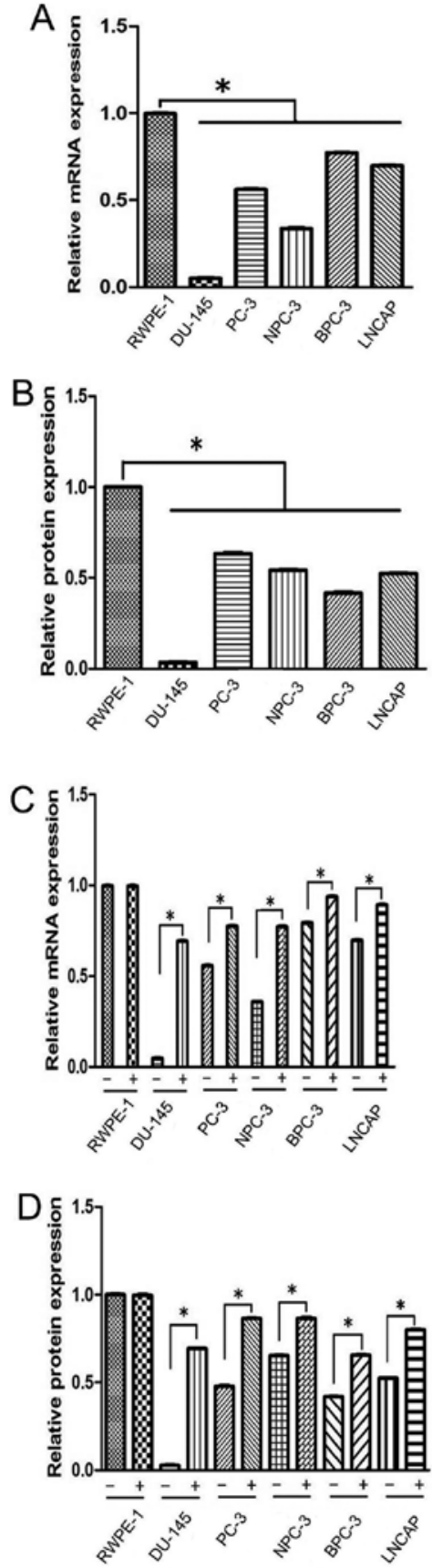

Figure 2. Expression levels of SPARC mRNA and protein. (A) As determined by qPCR, the mRNA expression level of SPARC was higher in the RWPE-1 cell line than in the other PCa cell lines. (B) The protein expression level of SPARC was higher in the RWPE-1 cell line than in the other PCa cell lines. (C) The mRNA expression levels of SPARC in PCa cell lines were increased after treatment with 5-Aza-Cdr. (D) The protein expression levels of SPARC in PCa cell lines were increased after treatment with 5-Aza-Cdr. ${ }^{*} \mathrm{P}<0.05$. SPARC, secreted protein acidic and rich in cysteine; $\mathrm{PCa}$, prostate cancer.

Investigation of SPARC gene hypermethylation. We further analyzed the hypermethylation status of the SPARC gene in PCa with the primers (Fig. 3) used in MSP, designed to detect hypermethylation of several CpG islands in the DU145, PC-3, NPC-3, BPC-3 and LNCAP cell lines.

Immunohistochemical analysis of the SPARC expression in PCa tissues and non-cancerous mucosa. To explore the expression and location of SPARC in tumors, immunos- 


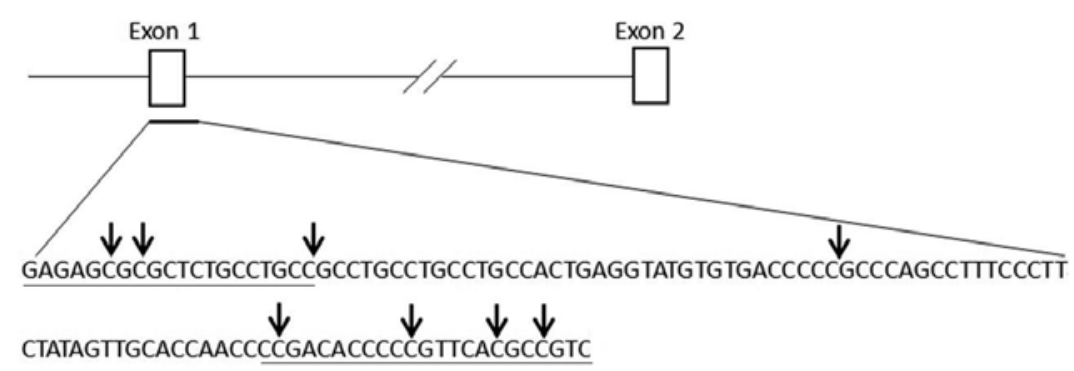

Figure 3. Illustration of the SPARC gene and topology of the MSP primer. CpG dinucleotides ' $\mathrm{C}$ ' in the objective sequence are marked by arrows. The underlined sequence indicates the primers for MSP. SPARC, secreted protein acidic and rich in cysteine; MSP, methylation specific PCR.
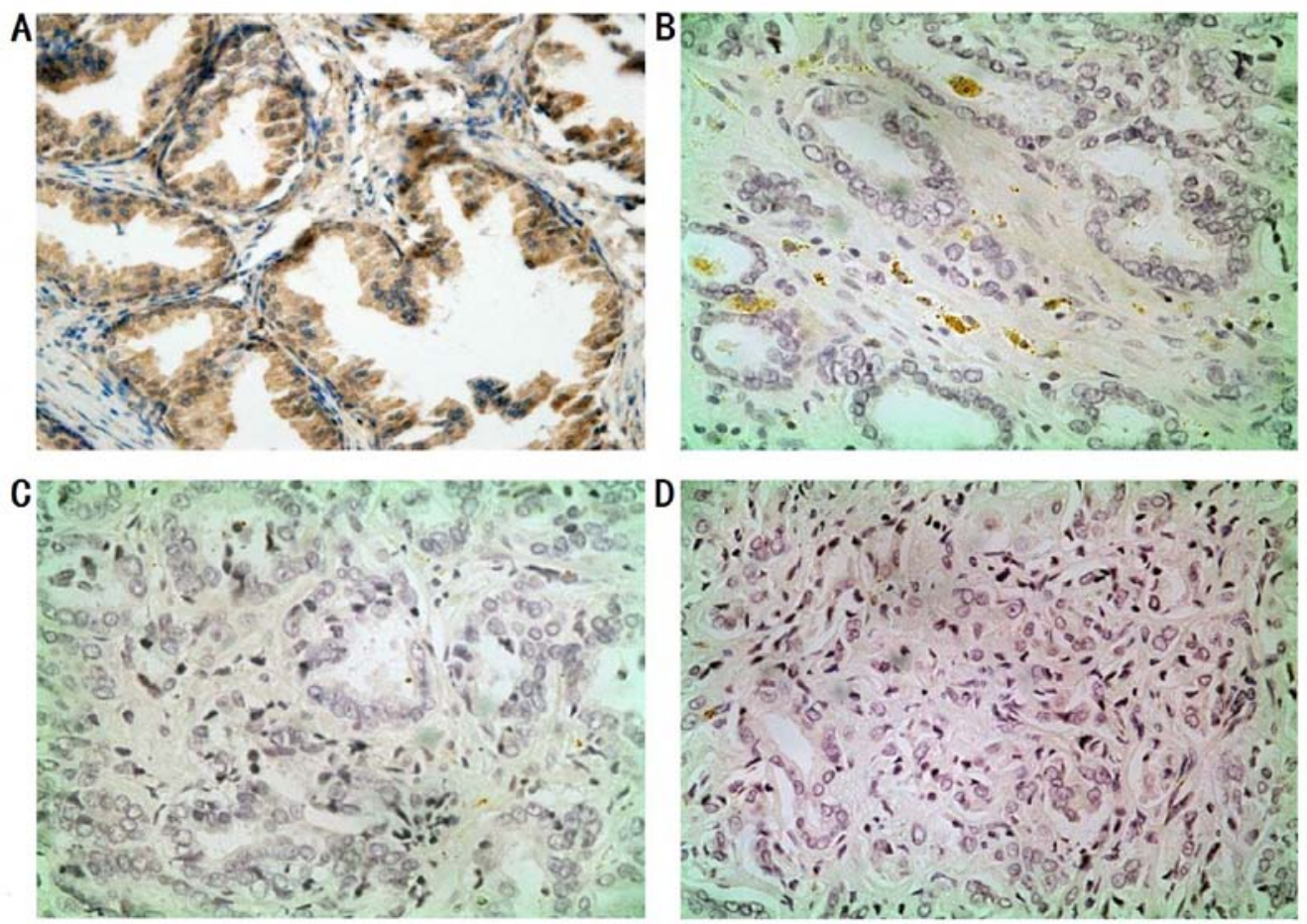

Figure 4. Immunohistochemical staining of the SPARC protein (x100 magnification). (A) Normal epithelial and stromal cells expressed a higher level of SPARC in the cytoplasm. (B) Immunostaining of SPARC was weak in well differentiated PCa cells. (C) Immunostaining of SPARC was faint or absent in moderately differentiated PCa cells. (D) Immunostaining of SPARC was absent in poorly differentiated diffused PCa cells. SPARC, secreted protein acidic and rich in cysteine; $\mathrm{PCa}$, prostate cancer.

taining was performed on PCa tissues and non-cancerous mucosa. We observed moderate (++) to strong (+++) SPARC expression in most stromal cells (80/108). In addition, we observed that the SPARC protein expression was closely associated with the degree of differentiation (Fig. 4). Weak and focal staining was also observed in the neoplastic epithelium $(28,25.9 \%)$. In the remaining cases $(80,74.1 \%)$, the neoplastic cells did not label for SPARC throughout the tumor.

5-Aza-Cdr inhibits cell proliferation, invasion and migration. As mentioned above, the hypermethylation of the SPARC promoter could be reversed following incubation with 5-Aza-Cdr, which also resulted in higher levels of SPARC mRNA and protein expression. To examine whether the alteration of SPARC levels in the PCa cells leads to changes in cell biological behaviors, we assessed cell proliferation, migration and invasion. The results indicated that 5-Aza-Cdr signifi- cantly decreased the proliferation, migration and invasion in the DU145 cell line (negative SPARC expression) compared to the PC-3 cell line (SPARC positive expression) (Fig. 5).

Correlation between SPARC hypermethylation and the clinicopathological parameters in PCa patients. We collected clinicopathological data from the patients and then analyzed the SPARC hypermethylation and the clinicopathological parameters in PCa patients. The results indicated hypermethylation of SPARC in prostate tumors (145 out of 207, 70\%), prostate mucosae (1 out of $38,2.6 \%$ ) and negative hypermethylation of SPARC in peripheral blood lymphocytes from patients ( 0 out of 30,0\%) and healthy volunteers (0 out of 30,0\%) (Fig. 6). The SPARC hypermethylation in the tumor tissues was not significantly associated with age, however, it was significantly associated with lymph node metastasis $(\mathrm{P}<0.001)$, advanced stages $(\mathrm{P}<0.001)$, a higher PI-RADS score $(\mathrm{P}=0.009)$ and a higher Gleason score $(\mathrm{P}<0.001)$ (Table I). 

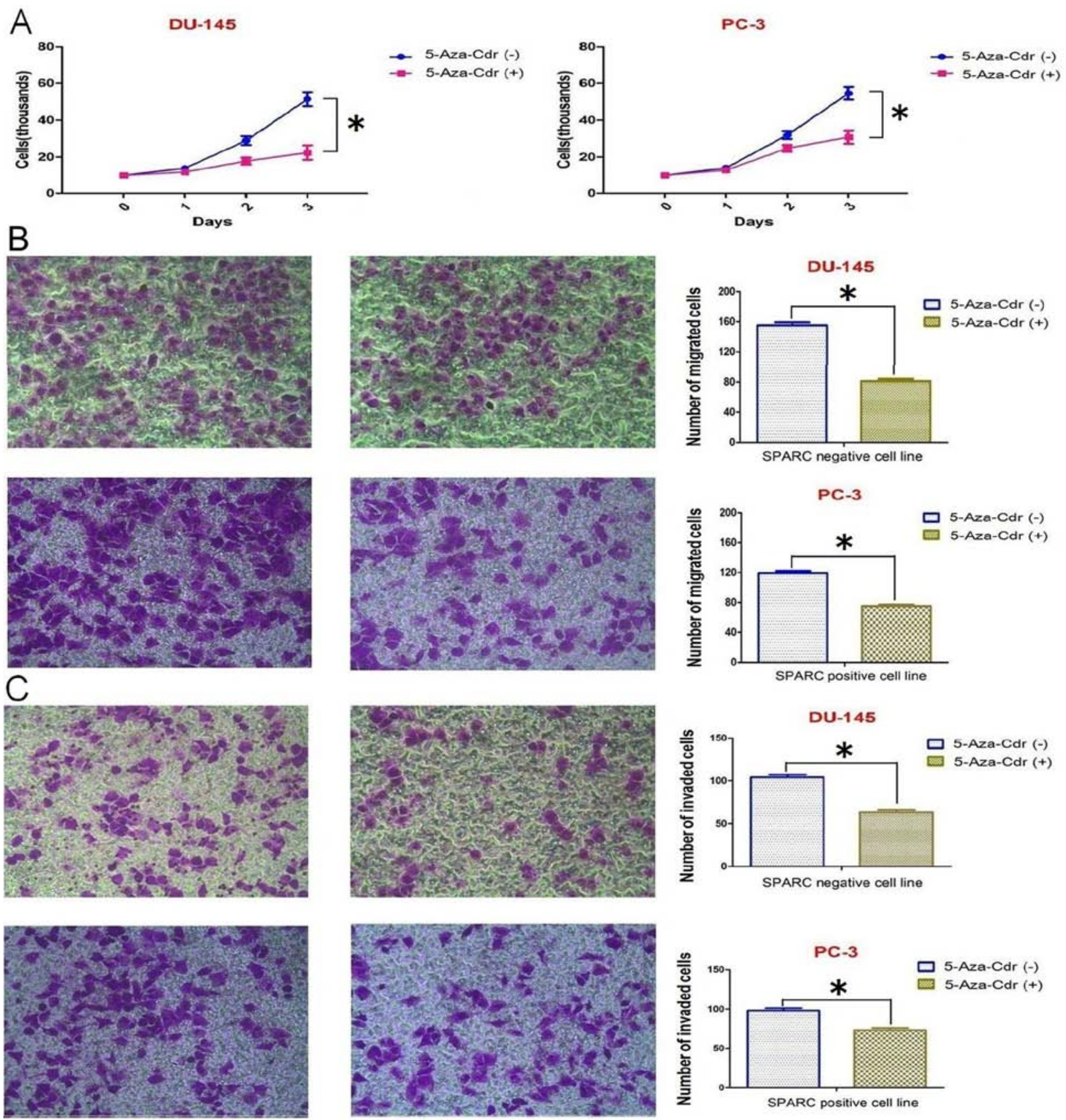

Figure 5. 5-Aza-Cdr treatment inhibits (A) proliferation, (B) migration and (C) invasion of DU145 (SPARC negative cell line) and PC-3 (SPARC positive cell line). ${ }^{*} \mathrm{P}<0.05$. SPARC, secreted protein acidic and rich in cysteine.

The overall survival curve revealed that patients with SPARC hypermethylation had a significantly poorer prognosis than those without SPARC hypermethylation $(\mathrm{P}=0.003$; log-rank test; Fig. 7), as well as for subgroups of patients at advanced stages $(\mathrm{P}=0.008)$, with higher PI-RADS score $(3,4,5)$, higher Gleason score $(8,9,10)(\mathrm{P}=0.025)$ and lymph node metastasis $(\mathrm{P}=0.016)$. In a multivariate Cox proportional hazards model, hypermethylation of the SPARC promoter was an independent adverse prognostic factor $[\mathrm{P}=0.005$; relative risk (RR) 2.659, 95\% CI, 1.433-4.562], as well as stage ( $\mathrm{P}=0.017$; RR 1.984, 95\% CI, 1.242-3.809), PI-RADS score ( $\mathrm{P}=0.013$, RR 3.781, 95\% CI, 1.474-6.237), Gleason score $(\mathrm{P}=0.032$; RR 3.545, 95\% CI, 1.246-5.619) and lymph node metastasis ( $\mathrm{P}=0.021$; RR $1.715,95 \% \mathrm{CI}, 2.052-5.751)$ in cancer cases (Table I).

\section{Discussion}

Prostate cancer is a heterogeneous malignancy with mostly unpredictable outcome. Currently, some clinicopathological features, such as PSA, Gleason score and clinical stages, are used to predict patient outcome in clinical practice. However, they fail to accurately distinguish aggressive tumors from non aggressive ones (24). Multiple studies indicated that aberrant DNA methylation is a hallmark of prostate cancer and is associated with malignant initiation and progression and demonstrated that aberrant DNA methylation may possibly be a potential biomarker of $\mathrm{PCa}(25)$.

In the present study, we investigated the role of DNA hypermethylation in the expression of SPARC in prostate cancer cells and stromal fibroblasts. In addition, we investigated the 
Table I. Correlation of the promoter hypermethylation of the SPARC gene with the clinical parameters of PCa patients.

\begin{tabular}{|c|c|c|c|c|c|c|c|c|}
\hline \multirow[b]{2}{*}{ Parameters } & \multirow{2}{*}{$\begin{array}{l}\text { No. }(\%) \\
(\mathrm{N}=207)\end{array}$} & \multirow{2}{*}{$\frac{\text { Hypermethylation }}{(\mathrm{N}=145)}$} & \multirow{2}{*}{$\frac{\text { Unmethylation }}{(\mathrm{N}=62)}$} & \multirow[b]{2}{*}{ P-value } & \multirow{2}{*}{$\frac{\text { Univariate }}{\text { P-value }}$} & \multicolumn{3}{|c|}{ Multivariate } \\
\hline & & & & & & Risk ratio & $95 \% \mathrm{CI}$ & P-value \\
\hline Age (years) & & & & $0.410^{\mathrm{b}}$ & 0.808 & 1.539 & $0.682-1.620$ & 0.786 \\
\hline$\geq 67^{\mathrm{a}}$ & $116(56.0)$ & $80(69.0)$ & $36(31.0)$ & & & & & \\
\hline$<67$ & $91(44.0)$ & $65(71.4)$ & $26(28.6)$ & & & & & \\
\hline PI-RADS score & & & & $0.009^{\mathrm{b}}$ & 0.010 & 3.781 & $1.474-6.237$ & 0.013 \\
\hline 1,2 & $17(8.2)$ & $7(64.7)$ & $10(35.3)$ & & & & & \\
\hline $3,4,5$ & $190(91.8)$ & $138(70.5)$ & $52(29.5)$ & & & & & \\
\hline TNM stage & & & & $<0.001^{\mathrm{b}}$ & 0.008 & 1.984 & $1.242-3.809$ & 0.017 \\
\hline I, II & $87(42.2)$ & $45(51.7)$ & $42(48.3)$ & & & & & \\
\hline III, IV & $120(57.8)$ & $100(83.3)$ & $20(16.7)$ & & & & & \\
\hline Gleason score & & & & $<0.001^{\mathrm{b}}$ & 0.025 & 3.545 & $1.246-5.619$ & 0.032 \\
\hline 7 & $68(32.9)$ & $37(54.4)$ & $31(45.6)$ & & & & & \\
\hline $8,9,10$ & $139(67.1)$ & $108(77.7)$ & $31(22.3)$ & & & & & \\
\hline Lymph node metastasis & & & & $<0.001^{\mathrm{b}}$ & 0.016 & 1.715 & $2.052-5.751$ & 0.021 \\
\hline Negative & 95 (45.9) & $52(54.7)$ & $43(45.3)$ & & & & & \\
\hline Positive & $112(54.1)$ & $93(83.0)$ & $19(17.0)$ & & & & & \\
\hline $\begin{array}{l}\text { Total SPARC } \\
\text { methylation } \\
\text { status }\end{array}$ & & $145(70.0)$ & $62(30.0)$ & & 0.003 & 2.659 & $1.433-4.562$ & 0.005 \\
\hline
\end{tabular}

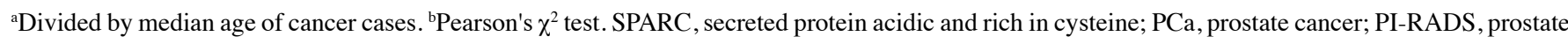
imaging reporting and data system; CI, confidence interval.

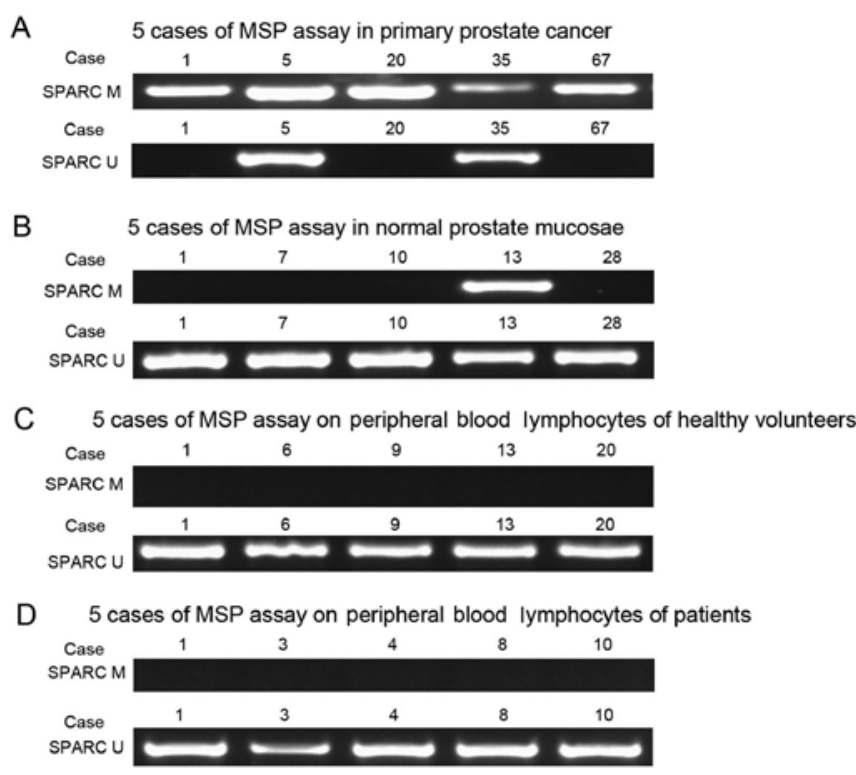

Figure 6. (A) Representative examples of MSP assay in primary tumors tissues. (B) Representative examples of the MSP assay in normal prostate mucosa samples. (C) Representative examples of the MSP assay in peripheral blood lymphocytes of healthy volunteers. (D) Representative examples of the MSP assay in peripheral blood lymphocytes of patients. The PCR products in the lanes $\mathrm{M}$ and $\mathrm{U}$ indicate the presence of hypermethylated and unmethylated fragments, respectively. MSP, methylation specific PCR.

correlation between SPARC hypermethylation status with clinicopathological parameters, as well as patient outcome.

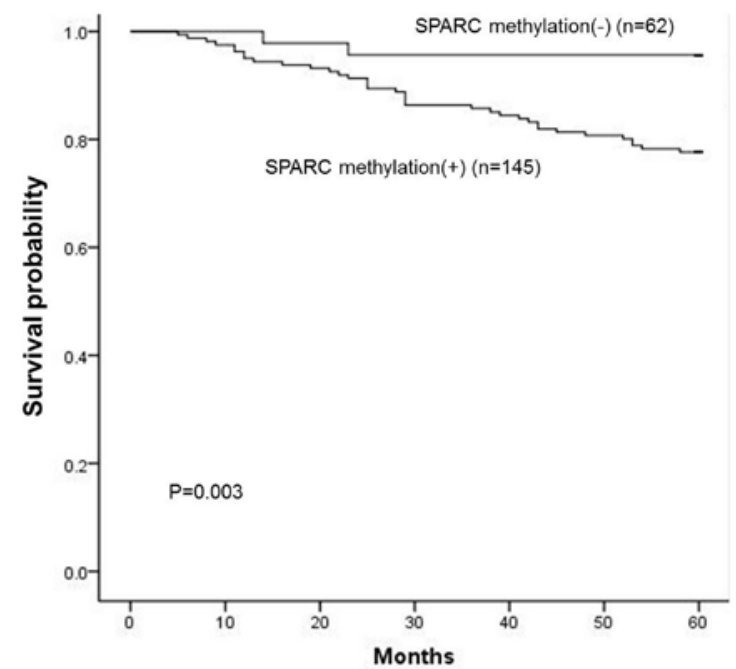

Figure 7. Kaplan-Meier analysis of overall survival for PCa patients with $(n=145)$ or without $(n=62)$ SPARC hypermethylation. Patients with SPARC hypermethylation in tumor tissue were closely correlated with poorer overall survival than patients without hypermethylation (Log-rank test, $\mathrm{P}=0.003$ ). $\mathrm{PCa}$, prostate cancer.

Our results revealed that hypermethylation of the SPARC promoter was the primary mechanism of SPARC downregulation. SPARC expression was frequently lost during the promoter hypermethylation but could be restored by 5-AzaCdr. In prostate cancer tissue, the change in the SPARC expression followed a similar pattern as in pancreatic cancer, 
where higher levels of SPARC were observed in normal epithelial cells whereas SPARC was absent in the majority of pancreatic cancer tissues (26). It is known that SPARC plays important roles in the development of proliferation and migration with complex biological effects and it is cell- and tumor-type specific (27). The loss of SPARC gene expression was also associated with promoter hypermethylation and could be rescued using 5-Aza-Cdr (23).

In PCa, although SPARC was reported as a predominantly protumorigenic protein (28), several studies revealed that SPARC downregulated the proliferation and invasion of $\mathrm{PCa}$ cells (12-14), indicating that SPARC was capable of becoming a useful immunohistochemical biomarker of PCa.

Our MSP results revealed that hypermethylation of specific $\mathrm{CpG}$ sites occurred at a high frequency in PCa cell lines, which could be a potential diagnostic or predictive marker of this disease. It is possible that the presence of complete or partial SPARC hypermethylation in some of these pathologically 'normal'-appearing epithelium may, in fact, represent an early epigenetic event in the adenocarcinoma sequence that predisposes these patients to develop prostate cancers. The confirmation of this hypothesis will require a further prospective study.

According to our data, advanced stage, higher Gleason score $(8,9,10)$, higher PI-RADS score $(3,4,5)$ and lymph node metastasis-positive were all associated with a poor prognosis in PCa. We also found that SPARC gene hypermethylation was correlated with a poor prognosis in PCa patients, which indicated that SPARC hypermethylation was associated with malignant behaviors of PCa cells.

Survival analysis indicated that patients with hypermethylated SPARC in the serum had worse overall survival than patients without methylated SPARC. Furthermore, SPARC hypermethylation was an independent predictive biomarker of poorer overall survival in PCa.

Our results also demonstrated that the SPARC expression was notably lower in cancer epithelial cells than that in stromal cells and 5-Aza-Cdr suppressed the biological behaviors of the prostate cell lines with SPARC promoter hypermethylation. A previous study (29) indicated that this inhibition may be associated with tumor growth and that SPARC had an antiproliferative function by modulating the cell cycle regulatory proteins or growth factors.

These results revealed that SPARC hypermethylation in serum is an independent prognostic biomarker in PCa. Patients with methylated SPARC in serum have higher risk of death. Therefore, it is a useful strategy to detect hypermethylated SPARC by using MSP on biopsies, serum and prostate lavage. Furthermore, SPARC hypermethylation profiling may be useful in establishing the epigenotype of each tumor in order to detect potential differences in occurrence of metastasis, sensitivity to chemotherapy and/or overall prognosis (28).

In conclusion, we demonstrated that SPARC expression was notably decreased in PCa mainly due to the hypermethylation of its promoter region. Furthermore, the downregulation of SPARC expression is necessary for the progression of $\mathrm{PCa}$ and the restored SPARC expression inhibited the proliferation, invasion and migration of prostate carcinoma cells. The hypermethylation of SPARC was closely associated with the prognosis of PCa. Therefore, the hypermethylation of SPARC presents a good predictive value for PCa survival.

\section{Acknowledgements}

The present study was supported by the National Nature Science Foundation of China (nos. 81602232, 81302542 and 81572519).

\section{References}

1. Siegel RL, Miller KD and Jemal A: Cancer statistics, 2015. CA Cancer J Clin 65: 5-29, 2015.

2. Siegel RL, Miller KD and Jemal A: Cancer Statistics, 2017. CA Cancer J Clin 67: 7-30, 2017.

3. Goessl C, Müller M, Heicappell R, Krause H, Straub B, Schrader M and Miller K: DNA-based detection of prostate cancer in urine after prostatic massage. Urology 58: 335-338, 2001.

4. Wang Y, Fan C, Yu J and Wang X: APC methylation predicts biochemical recurrence of patients with prostate cancer: A metaanalysis. Int J Clin Exp Med 8: 15575-15580, 2015.

5. Brocks D, Assenov Y, Minner S, Bogatyrova O, Simon R, Koop C, Oakes C, Zucknick M, Lipka DB, Weischenfeldt J, et al; ICGC Early Onset Prostate Cancer Project: Intratumor DNA methylation heterogeneity reflects clonal evolution in aggressive prostate cancer. Cell Reports 8: 798-806, 2014.

6. Hoque MO, Begum S, Topaloglu O, Jeronimo C, Mambo E, Westra WH, Califano JA and Sidransky D: Quantitative detection of promoter hypermethylation of multiple genes in the tumor, urine, and serum DNA of patients with renal cancer. Cancer Res 64: 5511-5517, 2004.

7. Bickers B and Aukim-Hastie C: New molecular biomarkers for the prognosis and management of prostate cancer--the post PSA era. Anticancer Res 29: 3289-3298, 2009.

8. Martignano F, Gurioli G, Salvi S, Calistri D, Costantini M, Gunelli R, De Giorgi U, Foca F and Casadio V: GSTP1 Methylation and protein expression in prostate cancer: Diagnostic implications. Dis Markers 2016: 4358292, 2016.

9. García-Tobilla P, Solórzano SR, Salido-Guadarrama I, González-Covarrubias V, Morales-Montor G, Díaz-Otañez CE and Rodríguez-Dorantes M: SFRP1 repression in prostate cancer is triggered by two different epigenetic mechanisms. Gene 593: 292-301, 2016.

10. Goltz D, Holmes EE, Gevensleben H, Sailer V, Dietrich J, Jung M, Röhler M, Meller S, Ellinger J, Kristiansen G, et al: CXCL12 promoter methylation and PD-L1 expression as prognostic biomarkers in prostate cancer patients. Oncotarget 7: 53309-53320, 2016.

11. Tai IT and Tang MJ: SPARC in cancer biology: Its role in cancer progression and potential for therapy. Drug Resist Updat 11: 231-246, 2008.

12. Kapinas K, Lowther KM, Kessler CB, Tilbury K, Lieberman JR, Tirnauer JS, Campagnola P and Delany AM: Bone matrix osteonectin limits prostate cancer cell growth and survival. Matrix Biol 31: 299-307, 2012.

13. Said N, Frierson HF Jr, Chernauskas D, Conaway M, Motamed K and Theodorescu D: The role of SPARC in the TRAMP model of prostate carcinogenesis and progression. Oncogene 28: 3487-3498, 2009.

14. Shin M, Mizokami A, Kim J, Ofude M, Konaka H, Kadono Y, Kitagawa Y, Miwa S, Kumaki M, Keller ET, et al: Exogenous SPARC suppresses proliferation and migration of prostate cancer by interacting with integrin $\beta 1$. Prostate 73: 1159-1170, 2013.

15. Wang Y, Yu Q, Cho AH, Rondeau G, Welsh J, Adamson E, Mercola D and McClelland M: Survey of differentially methylated promoters in prostate cancer cell lines. Neoplasia 7: 748-760, 2005.

16. Niskakoski A, Kaur S, Staff S, Renkonen-Sinisalo L, Lassus H, Järvinen HJ, Mecklin JP, Bützow R and Peltomäki P: Epigenetic analysis of sporadic and Lynch-associated ovarian cancers reveals histology-specific patterns of DNA methylation. Epigenetics 9: 1577-1587, 2014

17. Chen ZY, Zhang JL, Yao HX, Wang PY, Zhu J, Wang W, Wang X, Wan YL, Chen SW, Chen GW, et al: Aberrant methylation of the SPARC gene promoter and its clinical implication in gastric cancer. Sci Rep 4: 7035, 2014. 
18. Heitzer E, Artl M, Filipits M, Resel M, Graf R, Weißenbacher B Lax S, Gnant M, Wrba F, Greil R, et al: Differential survival trends of stage II colorectal cancer patients relate to promoter methylation status of PCDH10, SPARC, and UCHL1. Mod Pathol 27: 906-915, 2014.

19. Chen G, Tian X, Liu Z, Zhou S, Schmidt B, Henne-Bruns D, Bachem $M$ and Kornmann M: Inhibition of endogenous SPARC enhances pancreatic cancer cell growth: Modulation by FGFR1-III isoform expression. Br J Cancer 102: 188-195, 2010.

20. Tanpure S, Boyineini J, Gnanamony M, Antony R, Fernández KS Libes J, Lin J, Pinson D, Joseph PA and Gondi CS: SPARC overexpression suppresses radiation-induced HSP27 and induces the collapse of mitochondrial $\Delta \psi$ in neuroblastoma cells. Oncol Lett 13: 4602-4610, 2017.

21. Kordi-Tamandani DM, Moazeni-Roodi AK, Rigi-Ladiz MA, Hashemi M, Birjandian E and Torkamanzehi A: Promoter hypermethylation and expression profile of MGMT and CDH1 genes in oral cavity cancer. Arch Oral Biol 55: 809-814, 2010.

22. Herman JG, Graff JR, Myöhänen S, Nelkin BD and Baylin SB: Methylation-specific PCR: A novel PCR assay for methylation status of CpG islands. Proc Natl Acad Sci USA 93: 9821-9826, 1996.

23. Socha MJ, Said N, Dai Y, Kwong J, Ramalingam P, Trieu V, Desai N, Mok SC and Motamed K: Aberrant promoter methylation of SPARC in ovarian cancer. Neoplasia 11: 126-135, 2009.
24. Kristensen H,Haldrup C, Strand S, Mundbjerg K, Mortensen MM, Thorsen K, Ostenfeld MS, Wild PJ, Arsov C, Goering W, et al: Hypermethylation of the GABRE miR-452 miR-224 promoter in prostate cancer predicts biochemical recurrence after radical prostatectomy. Clin Cancer Res 20: 2169-2181, 2014.

25. Strand SH, Orntoft TF and Sorensen KD: Prognostic DNA methylation markers for prostate cancer. Int J Mol Sci 15: 16544-16576, 2014.

26. Gao J, Song J, Huang H, Li Z, Du Y, Cao J, Li M, Lv S, Lin H and Gong Y: Methylation of the SPARC gene promoter and its clinical implication in pancreatic cancer. J Exp Clin Cancer Res 29: $28,2010$.

27. Podhajcer OL, Benedetti LG, Girotti MR, Prada F, Salvatierra E and Llera AS: The role of the matricellular protein SPARC in the dynamic interaction between the tumor and the host. Cancer Metastasis Rev 27: 691-705, 2008.

28. Jacob K, Webber M, Benayahu D and Kleinman HK: Osteonectin promotes prostate cancer cell migration and invasion: A possible mechanism for metastasis to bone. Cancer Res 59: 4453-4457, 1999.

29. Yang E, Kang HJ, Koh KH, Rhee H, Kim NK and Kim H: Frequent inactivation of SPARC by promoter hypermethylation in colon cancers. Int J Cancer 121: 567-575, 2007. 